\title{
Belgeo
}

Revue belge de géographie

$4 \mid 2013$

Miscellaneous

\section{Les Coréens aux Philippines, jeux d'échelles et mobilités}

Koreans in The Philippines, effects of scale and mobilities

\section{Catherine Guéguen}

\section{(2) OpenEdition}

\section{Journals}

\section{Édition électronique}

URL : http://journals.openedition.org/belgeo/11584

DOI : 10.4000/belgeo.11584

ISSN : 2294-9135

Éditeur :

National Committee of Geography of Belgium, Société Royale Belge de Géographie

\section{Édition imprimée}

Date de publication : 31 décembre 2013

ISSN : 1377-2368

\section{Référence électronique}

Catherine Guéguen, « Les Coréens aux Philippines, jeux d'échelles et mobilités », Belgeo [En ligne], 4 | 2013, mis en ligne le 24 juin 2014, consulté le 19 avril 2019. URL : http://journals.openedition.org/ belgeo/11584; DOI : 10.4000/belgeo.11584

Ce document a été généré automatiquement le 19 avril 2019

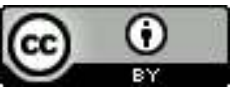

Belgeo est mis à disposition selon les termes de la licence Creative Commons Attribution 4.0 International. 


\title{
Les Coréens aux Philippines, jeux d'échelles et mobilités
}

\author{
Koreans in The Philippines, effects of scale and mobilities
}

\section{Catherine Guéguen}

\section{Introduction}

1 Les populations coréennes installées en dehors de leur péninsule représentent environ 7 millions de personnes en 2013. Le profil des migrants coréens a changé depuis les années 1960 avec le développement économique du pays. Le gouvernement adopta aussi une importante politique d'émigration, laquelle participait au contrôle de la population. Les candidats au départ s'orientaient d'abord massivement vers des pays plus industrialisés et principalement les Etats-Unis. Mais la communauté coréenne en Asie s'élève désormais à 3,9 millions de personnes, tandis qu'en Amérique du nord l'effectif est de 2,4 millions, l'Europe quant à elle compte 615000 Coréens. Outre la stabilité voire l'augmentation des effectifs dans les grandes zones d'accueil, les flux migratoires se recomposent et ce surtout à l'échelle du continent asiatique. En effet, les bouleversements géopolitiques, économiques et les accords de coopération récents génèrent des réorientations des flux de migrants coréens, lesquels jouent des nouvelles opportunités et des territoires pour accroître leur potentiel mobilitaire.

2 La diversification de l'implantation des Coréens outre-mer et sa compréhension attirent toute notre attention. Leur attraction pour les Philippines en particulier amène de multiples interrogations. En effet, leur présence est véritablement le résultat d'une mise en contact de deux espaces aux caractéristiques bien différentes et d'initiatives à la fois institutionnelles, inscrites à différentes échelles, mais aussi individuelles au vu des profils des migrants coréens. L'autre intérêt de l'étude repose sur le profil des Philippines, pays largement au cœur de la géographie des flux de populations et souvent étudié comme tel, mais qui est désormais un pays d'immigration. 
3 Les Philippines sont au cœur de la géographie des flux de populations par la très forte émigration organisée par l'Etat. On dénombre environ dix millions de Philippins vivant en dehors de leur pays-archipel. Ces migrations furent initiées sous la présidence Marcos dans les années soixante-dix. Ce phénomène, y compris les impacts de cette ponction démographique, a largement été étudié (Parreñas, 2005). Les retours d'argent de cette main d'œuvre constituent encore aujourd'hui près de $20 \%$ des revenus de l'Etat philippin. Cette capacité de formation à la migration est désormais mise à disposition de migrants coréens, et les Philippines, forte de 92,5 millions d'habitants (NSO, mai 2010) constituent une intéressante zone de débouchés commerciaux. Ainsi, les Philippines accueillent désormais de nouvelles vagues de populations de Corée du sud. La visibilité des migrants et visiteurs coréens est plus marquée depuis cinq ans.

4 Les données statistiques dénombrant les Coréens sur le sol philippin sont relativement opaques. Deux sources permettent toutefois le dénombrement : le National Statistics Office qui, à partir de recensements décennaux, évalue le nombre d'étrangers sur le sol philippin. L'autre source est le Bureau de l'Immigration qui comptabilise les émissions de visas (étude, professionnel..). On ne peut toutefois considérer ces données que comme indicatives puisque de nombreux étrangers vivent sur le sol philippin sans la documentation nécessaire. Le ministère des affaires étrangères coréen indique ainsi pour l'année 2009, 115400 Sud-Coréens installés aux Philippines mais il l'évalue à 88102 en 2013. Ils représentent $1,6 \%$ des 7 millions de Sud-Coréens installés à l'étranger. En revanche, le nombre de touristes coréens aux Philippines progresse constamment: ils étaient 600000 en 2008, ils étaient déjà 800000 à se rendre dans l'archipel de janvier à août 2013 (soit 120000 plus sur la même période qu'un an auparavant) ${ }^{1}$. L'objectif des 1,4 million de visiteurs est clairement avancé par le Ministère du Tourisme philippin (DOT: Department of Tourism). Ces flux de populations s'inscrivent aussi dans un nouveau contexte : les Philippines maintiennent une croissance économique de $3 \%$ à $6 \%$ depuis 2010 et misent sur la demande de proximité des Etats voisins pour intensifier les mobilités touristiques qui touchent l'archipel. Il en va également du changement de l'image touristique du pays qui passe par une reconfiguration des activités de ce secteur longtemps orienté «de manière déguisée » vers le tourisme du sexe, sous forme de voyages d'affaires masculins organisés à partir du Japon par exemple. Les interactions entre communautés coréennes installées de manière pérenne et celles qui le sont de manière temporaire méritent qu'on s'y intéresse. On exclura de l'étude les Coréens expatriés travaillant pour de grandes entreprises internationales, lesquelles participent peu à ces interactions.

5 En quoi la création d'un nouvel espace de mobilités en Asie du sud-est, intégrant les pays émergents de la zone dont la Corée du sud, renforce-t-elle le rôle des communautés résidentes coréennes et celui de l'Etat philippin dans la consolidation de ces flux de populations?

6 L'espace de circulation comprenant les différents pays de l'ASEAN et leurs associés dont la Corée du sud, constitue une aire d'expansion économique mais dont les niveaux de vie nationaux sont très disparates. Jouant de cette différence, les Philippines ont su attirer des populations en quête de formation ou d'expérience professionnelle à l'étranger. Les liens entre communautés coréennes résidantes et touristes s'inscrivent dans des lieux spécifiques liés à une offre de formation. L'étude s'inscrit donc dans un espace de mobilité régionale mais les études de cas aux échelles locales voire intra-urbaines permettent une 
mise en perspective du fonctionnement de ces communautés coréennes plurielles aux Philippines.

\section{La présence coréenne aux Philippines : un front pionnier mobilitaire?}

\section{Vers une diversification des implantations coréennes en Asie}

7 La géographie des foyers de populations coréennes installées outre-mer indique une forte concentration des effectifs : $80 \%$ des Coréens outre-mer sont installés dans trois pays (les Etats-Unis, la Chine et le Japon) . Pourtant, cinq Etats en accueillent plus de 100000 et 15 au moins 10000. Les pays d'implantation des Coréens évoluent et leur engouement rapide pour la Nouvelle-Zélande peut surprendre : ils n'étaient que 3000 en 1992, atteignaient les 18000 en 2001 et sont désormais plus de 30 000. Au-delà des données statistiques souvent différentes entre le pays de départ et les pays d'accueil, ces dernières indiquent nettement des zones d'accueil et des communautés coréennes parfois importantes qui n'ont été que peu étudiées ou pas du tout comme celle du Japon, comme le soulignent Ryang S. \& Lie J. (2009).

Plus de 50 \% des Coréens installés à l'étranger le sont en Asie orientale. Ils se sont orientés massivement vers le Japon, pays en pleine ascension économique dans les années 1960 où ils étaient déjà 400000 . Ce flux migratoire réamorcé vers le Japon rappelle que de nombreux Coréens y étaient déjà présents depuis la période coloniale japonaise durant laquelle ils s'étaient installés pour formation dans le cadre de l'empire japonais (Tugault, 1984). La forte présence coréenne en Chine fut aussi constituée en plusieurs étapes; les Coréens étaient déjà présents dans les zones frontalières chinoises avant la colonisation japonaise du début du XXème siècle. Par la suite, avec la mise en valeur de ces zones minières, des transferts de populations coréennes ont été opérés par l'Etat colonial japonais. Enfin, les trente dernières années ont vu un afflux récent et renouvelé de populations coréennes sur le sol chinois : les espaces de la présence coréenne actuelle ne correspondent pas forcément aux lieux d'implantation plus anciens. Ce sont exclusivement des Sud-Coréens qui participent à ces nouvelles mobilités liées aux facilités de circulation et aux possibilités de séjour de longue durée en Chine pour affaire. Leur présence aux Philippines s'est largement accentuée depuis 2005 avec l'intégration de la Corée du sud dans l'espace de mobilité de l'ASEAN.

\section{La Corée du sud intégrée dans la zone de mobilité de l'ASEAN}

Les flux de populations récents de Corée du sud accompagnent une dynamique d'ouverture dans le cadre de l'ASEAN mais aussi de la part des Philippines. En effet, des liens anciens unissent ces deux Etats mais ils ne s'étaient pas traduits par des vagues migratoires. L'émigration sud-coréenne fut favorisée par l'Etat coréen à partir de 1962 pour gagner des devises étrangères (flux orientés vers l'Amérique du nord et l'Amérique latine). Ce n'est qu'à partir de la fin des années 1980 que les trajectoires se diversifient et ce en parallèle avec l'amélioration du niveau de vie en Corée du sud. Ces flux de populations accompagnent l'expansion des entreprises coréennes. Les relations entre les Philippines et la Corée du sud sont régulées par les accords ASEAN + 3, dans lesquels sont aussi intégrés le Japon et la Chine. L'ASEAN et la Corée du sud avaient initié des 
discussions depuis novembre 1989. Les accords entre les deux Etats sont orientés vers des questions politiques et de sécurité, mais aussi de coopération économique accentuée depuis 2005. Les relations socio-culturelles sont aussi encouragées tout comme des projets de coopération entre les membres de l'ASEAN et la Corée du sud depuis 1990, lesquels ont été renouvelés en 1997. Les liens entre les deux Etats sont renforcés par un passé «commun»: celui de la présence américaine, qui a renforcé une volonté de modernité à l'occidentale.

L'élévation du niveau de vie en Corée du sud guide ces migrants en quête d'un mode de vie « à l'occidentale » au début des années 1990. L'arrivée récente des étudiants coréens aux Philippines est révélatrice de la constitution d'une classe moyenne nationale, qui recherche des formations de qualité et anglophones; ils n'ont pas les moyens d'aller étudier en Amérique du nord ou en Australie. Les Philippines deviennent au cours de la décennie suivante une destination bon marché pour profiter du golf et des plages. L'accentuation des flux de populations entre les deux pays traduit aussi la mise en place d'un espace touristique asiatique. $50 \%$ des touristes en Asie orientale sont des Asiatiques (P. Pelletier, 2012), le tout dépendant du niveau de développement des pays émetteurs. Ainsi, les Japonais sont de longue date les visiteurs les plus nombreux dans pratiquement tous les pays de la région ( $15 \%$ de la clientèle en moyenne). Les ressortissants chinois, auxquels les visas touristiques sont accordés plus libéralement, constituent le second groupe de touristes internationaux en Asie (le premier en Asie du sud-est). Cet espace structuré par les flux touristiques à partir du Japon et des pays émergents de la zone constitue le troisième bassin touristique mondial après la Méditerranée et le bassin caribéen. Manille cherche ainsi à densifier son activité touristique en jouant sur des complémentarités de compétences, et les évolutions du transport aérien tendent à faire de la région capitale des Philippines un hub régional captant les clientèles de la Chine littorale ou des autres capitales asiatiques.

\section{Une offre en transport améliorée à l'initiative des Philippines}

11 La massification des mobilités dans l'espace de l'ASEAN a aussi été déterminée par la densification du transport aérien entre Manille et la Corée du sud. Avec des tarifs défiant toute concurrence, les migrants coréens circulent très aisément dans l'espace de l'Association des nations d'Asie du sud-est. La capitale, Manille, a étendu ses infrastructures aéroportuaires avec la mise en service depuis 2007 de l'aéroport NAIA III, mis à la disposition des compagnies aériennes Cebu Pacific et Philippines Airlines pour leurs destinations nationales et internationales. L'offre aéroportuaire s'est également élargie avec la mise en service de l'aéroport de Clark, aménagé dans l'ancienne base américaine localisée à une centaine de kilomètres de Manille. A titre d'exemple, la compagnie philippine Cebu Pacific dessert Seoul-Incheon et Pusan à partir de Manille et Cebu. La compagnie Philippine Airlines effectue aussi des rotations sur la Corée en desservant SeoulIncheon à partir de Manille et Cebu. Par semaine, une cinquantaine de vols relient les Philippines à la Corée et, ainsi, environ 8000 personnes circulent entre les deux pays. La durée du vol entre la Corée du sud et les Philippines est d'environ $3 \mathrm{~h} 30$ (sans décalage horaire). Depuis 2012, la compagnie Zest air repositionne ses vols à l'international vers des destinations prisées par les Coréens comme Manille, Clark et Cebu mais elle ajoute à ses services la desserte de «la» niche touristique philippine: l'île de Boracay dans les Visayas. Elle propose désormais des vols à partir d'Incheon-Seoul desservant Kalibo, la 
ville la plus proche de Boracay, dont l'aéroport peut accueillir des longs courriers. Cette compagnie capte non seulement les Coréens du sud mais aussi les Chinois de Shanghai selon le même principe. Capter cette population de classe moyenne fait partie des stratégies mises en place par cette compagnie aérienne qui fonctionne en charter à partir de la Corée du sud et par association avec des promoteurs touristiques et immobiliers philippins et coréens. Le relais entre les initiatives philippines et les touristes coréens est assuré par une population résidante coréenne dans les lieux qu'ils fréquentent (figure 1).

\section{Des conditions de résidence facilitées par les autorités philippines}

12 L'espace de l'ASEAN constitue un espace d'échanges régulés pour les marchandises mais relativement souples concernant la circulation des hommes. Dans le cadre des accords multilatéraux avec les pays de l'ASEAN, les citoyens coréens circulent plus facilement; ces derniers disposent d'un visa touristique de trente jours ${ }^{2}$ à leur entrée sur le sol philippin. Ce visa est délivré à leur arrivée dans un des quatre aéroports internationaux philippins (Manille, Cebu, Davao et Kalibo depuis 2012). Aux Philippines, n'importe quel «touriste" peut prolonger son séjour en étendant son visa moyennant quelques centaines de pesos dans les locaux de l'Immigration présents dans les grandes villes ou zones portuaires. Certains d'entre-eux sont spécialisés auprès de certaines communautés, par exemple un agent administratif coréen prête main forte aux employés du Bureau de l'immigration de Baguio pour faciliter la traduction; ce dernier a été dépêché sur place à la demande des associations coréennes de la ville.

Hormis le visa de tourisme délivré à l'arrivée, les Coréens peuvent faire la demande auprès des services consulaires philippins en Corée de visa de longue durée. Les consulats et Bureaux de l'immigration proposent des visas de résidence spécifique pour retraité ou entrepreneur. En effet, le SRR Visa (Special Resident Retiree's Visa) permettent l'octroi d'un visa de longue durée, qui implique également une disponibilité numéraire placée sur un compte bancaire et diverses démarches administratives. Il leur est interdit d'exercer une activité professionnelle. Dans le cas des Coréens, l'attractivité réside dans les coûts de résidence et de soins moindres que dans leurs pays d'origine. Les deux autres types de visa longue durée impliquent une activité salariée. Le visa d'investissement autorise un homme d'affaires à établir une activité quelconque, généralement d'import-export entre son pays d'origine et les Philippines : 50000 USD sont nécessaires pour l'établissement de ces formalités. Plus récemment l'Etat philippin a crée le SVEG, c'est à dire le Special Visa for Employment Generation. Ce visa n'est délivré que dans le cas de la création d'au moins dix emplois aux Philippines, et ce, après l'aval des différentes administrations du travail, de la santé et de la sécurité sociale. Créé en 2009, ce dernier semble être le moyen le plus légal de rester aux Philippines sans utiliser de prête-noms ou de contracter un mariage blanc. L'opacité des données accompagne toutefois la résidence illégale de nombreux Coréens, laquelle est permise par la corruption locale. 
Figure 1. Commerce coréen à Baguio faisant la promotion du SVEG permettant l'octroi d'un permis de résidence aux Philippines par la création d'emplois (août 2010).

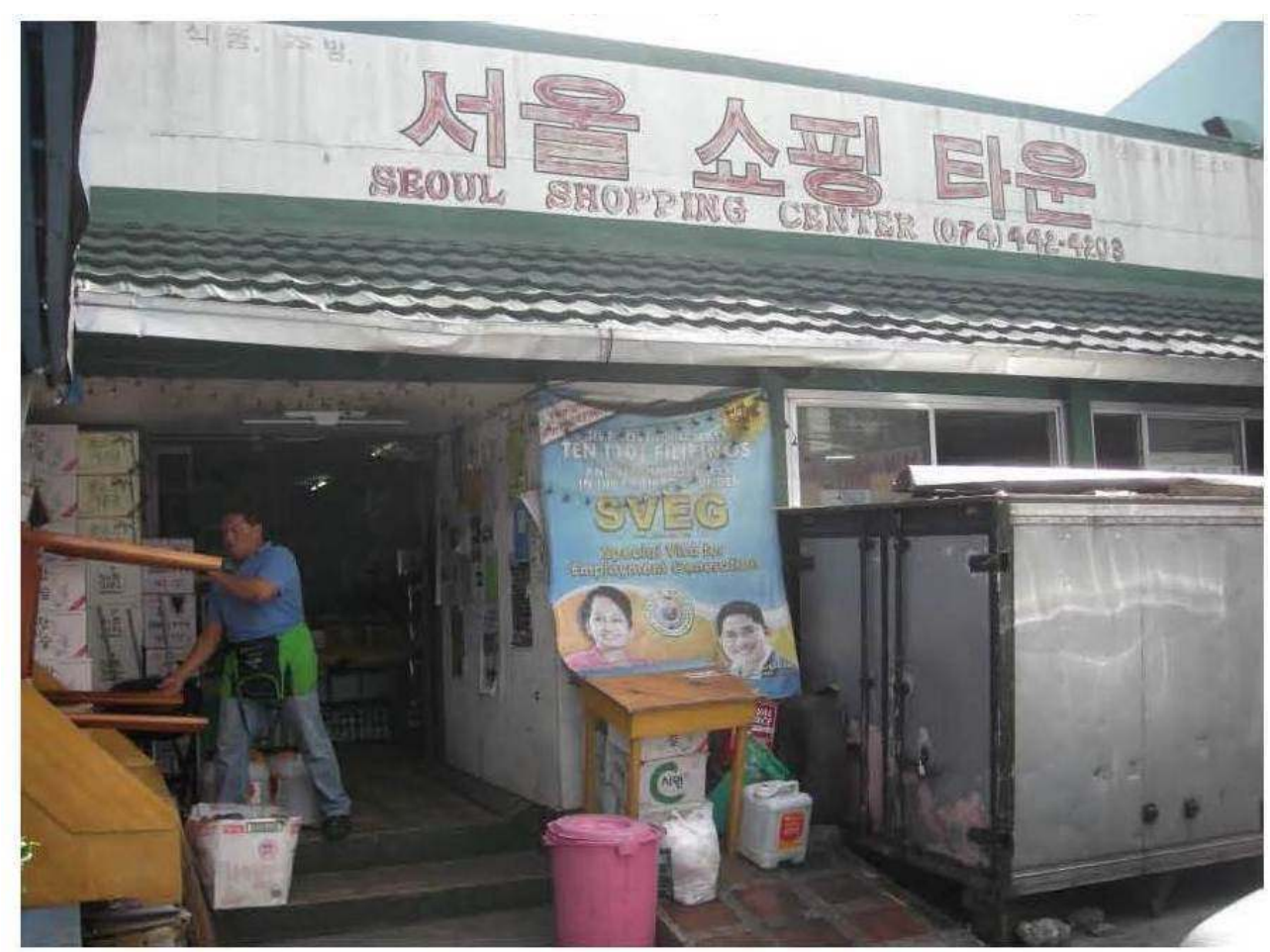

14 Les liens économiques axés sur les échanges de produits manufacturés ont généré de nouvelles vagues migratoires de populations coréennes vers les Philippines. Malgré l'évidence de ce constat, les situations sont variées. Certaines migrations peuvent s'apparenter à des migrations économiques «inversées ", dans le sens où le migrant est originaire d'une zone plus développée que celle de son pays d'accueil. Ces migrations prennent un ton particulier dans la capitale philippine. Les Coréens sont investis dans l'import-export ou dans la vente de matériel informatique d'occasion. Ces migrants visent les populations pauvres locales; ce sont pour la plupart des indépendants ayant perdu leur emploi en Corée. Ils s'investissent aussi parfois dans des centres linguistiques « temporaires ». De nombreux Coréens résident à la limite de la légalité aux Philippines et n'hésitent pas à "souscrire » un mariage blanc ou à utiliser un prête-nom pour ouvrir une affaire.

Quelques-uns travaillent pour le compte de sociétés coréennes ayant délocalisé une entreprise aux Philippines; ces dernières sont principalement implantées au sud de Manille dans les provinces de Laguna, Cavite ou Batangas. Ces expatriés coréens constituent une exception, puisque dans la plupart des cas, les Coréens investis dans le commerce s'intéressent au marché des populations pauvres locales ou à leurs compatriotes séjournant aux Philippines pour de courts séjours. 
Figure 2. Points d'entrée et lieux privilégiés des Sud-Coréens aux Philippines.

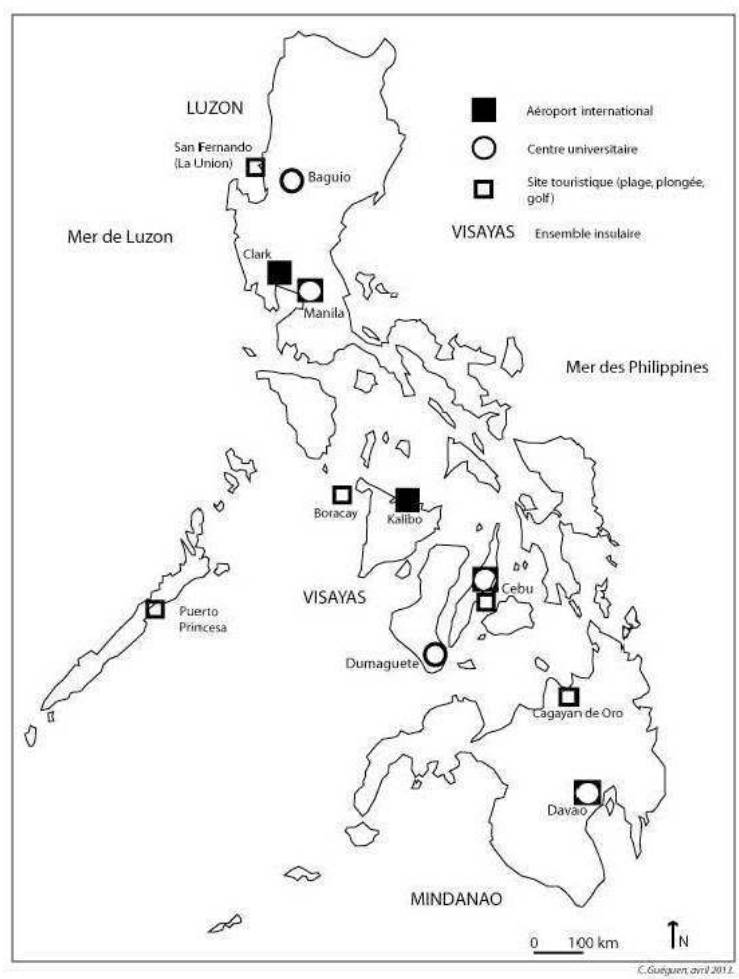

Les dispositifs institutionnels et de promotion de l'archipel philippin en Corée du sud menés activement depuis quelques années portent leurs fruits; les Coréens sont désormais les premiers visiteurs des Philippines : leur nombre atteignait 925204 en 2011 soit $23 \%$ des arrivées (à titre de comparaison, en 2011, les Japonais n'étaient que 375496 à se rendre dans l'archipel philippin). L'objectif de 1,4 million de touristes coréens visé par le Ministère du tourisme en 2013 semble réalisable. L'attractivité des Philippines pour la classe moyenne coréenne réside également dans la participation indispensable des Coréens installés sur place dans l'organisation de ces mobilités touristiques.

\section{Lieux et effets d'agrégation de cette mobilité sud- coréenne aux Philippines}

\section{Là où il y a déjà des Coréens}

Les Coréens sont connus pour évoluer en "petits groupes » dans la capitale du pays mais leur présence se renforce dans les grandes villes de province, plus particulièrement à Dumaguete et Cebu dans les Visayas, Davao sur l'île de Mindanao et Baguio au nord de Manille. Dans Metro-Manila, les Coréens sont plutôt dispersés et investissent les lieux d'éducation.

Excepté des regroupements de populations coréennes liés à des implantations industrielles, comme l'entreprise coréenne Hanjin à Subic, les Coréens du sud s'intègrent dans le tissu urbain existant. On les retrouve dans les lieux associant la proximité des lieux de travail et des zones résidentielles confortables. De petits groupes de Coréens se rassemblent dans des subdivisions résidentielles du sud de Metro-Manila (Paranaque, Las 
Pinas), lesquelles permettent grâce à l'autoroute reliant le sud de Luzon (South Express Way) de rallier les zones industrielles de Batangas.

L. Dehoorne (2002) cite l'exemple des Coréens en établissant les logiques des flux touristiques et les territoires migratoires. Il insiste sur le fait que peu de communautés sont concernées de façon significative par ces interactions tourisme-diaspora. Les mobilités touristiques des Sud-Coréens sont associées à des services marchands et privilégient les ressortissants de leur communauté lors des voyages à l'étranger. Les SudCoréens n'ont commencé à expérimenter les voyages touristiques à l'étranger que depuis la fin des années 1980. Pour organiser leurs séjours, ils privilégient les services d'agences nationales travaillant avec des ressortissants établis dans les villes des pays visités. Les membres de la communauté à l'étranger commercialisent des chambres chez l'habitant, tiennent des restaurants coréens, ce qui traduit un fonctionnement communautaire. Les difficultés linguistiques impliquent aussi ce rapprochement. Si Joong Kim (2003, p. 213) souligne aussi cet effet d'agrégation concernant les communautés coréennes dans les grandes villes chinoises : Beijing, Tianjin et Shanghai, qui attirent ces nouveaux migrants coréens travaillant au service de leurs communautés dans les Korea towns à l'image de Wangjing au Nord-est de Beijing où résident 10000 Coréens.

Ainsi, l'offre touristique des membres de la communauté coréenne à l'étranger est exclusivement orientée vers les nationaux (via les agences et des annonces rédigées en coréen), laquelle est particulièrement remarquable dans les lieux touristiques internationaux comme l'île de Boracay. Les centres de formation linguistique restent très discrets dans le paysage urbain avec peu ou pas de signalétiques; les nouveaux centres sont construits en périphérie de la ville comme à Baguio et les étudiants coréens évoluent quasiment en vase clos. Toutes les activités jouant sur les compétences de l'archipel, que ce soit au niveau du tourisme ou des cours de langue, sont organisées par des Coréens résidant aux Philippines. Sur l'île de Boracay, les Coréens constituent le premier groupe de visiteurs internationaux dans l'île selon les statistiques réalisées par le département du tourisme de la région VI avec une fréquentation comprise entre 11000 et 15000 visiteurs selon les mois ${ }^{3}$. Des structures hôtelières de grande taille privilégient l'accueil de groupes de Coréens auxquels elles proposent des tarifs compétitifs afin d'avoir un taux de remplissage maximal tout au long de l'année ${ }^{4}$. La pérennité et l'accentuation de ces flux de touristes coréens sur l'île de Boracay renforcent la présence de Coréens résidant sur place: des guides touristiques, des pasteurs, des commerçants, lesquels constituent un socle pour réceptionner cette population de visiteurs temporaires. Preuve de l'importance de leur présence sur place, ils disposent de quelques pages dans le journal local de la communauté des expatriés sur l'île, laquelle fut longtemps dominée par des Anglo-saxons puis relayée par les Coréens et les Russes. 
Figure 3. Eglise coréenne et autres services dédiés aux estivants coréens au rez-de-chaussée île de Boracay (février 2013).

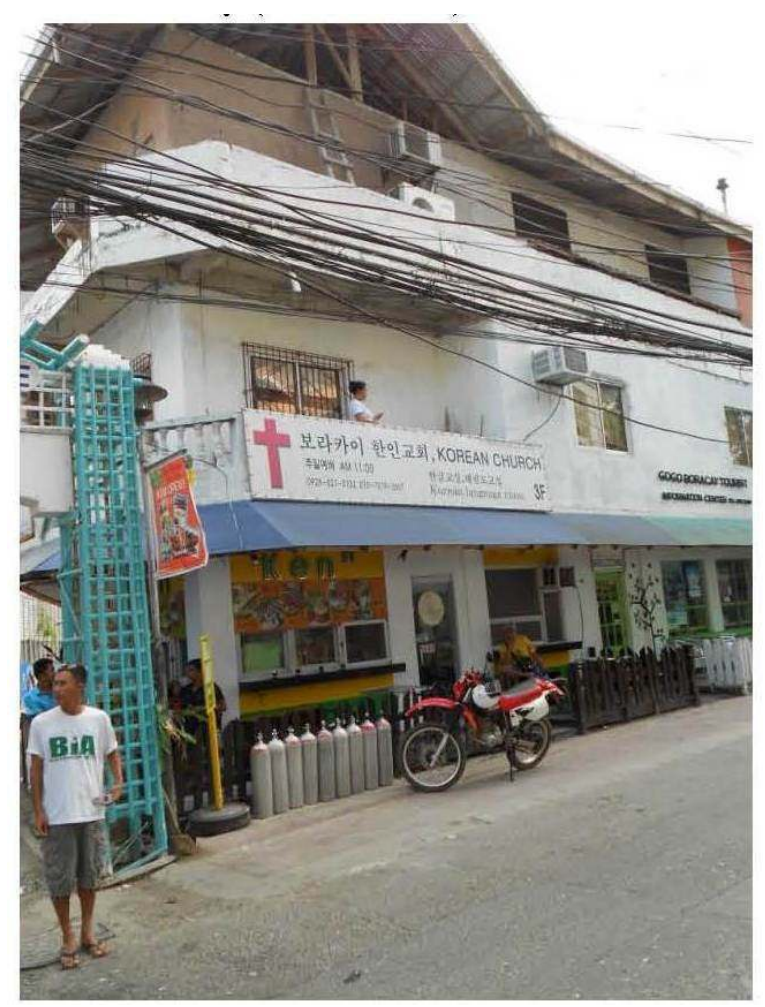

\section{Le choix des quartiers résidentiels}

Dans les agglomérations, les quartiers dans lesquels résident les Coréens ont des spécificités communes. En effet, qu'il s'agisse d'une localisation dans l'agglomération capitale ou dans les villes de province, les quartiers résidentiels sont généralement de qualité. Les Coréens habitent dans des espaces dédiés à la location, composés de maisons individuelles (appelées «bungalow» sur place) ou parfois des maisons mitoyennes («townhouses») dans des subdivisions résidentielles "à l'américaine ». Ces propriétés disposent d'un jardin de taille modeste et font généralement partie d'un quartier fermé ou « village » si bien qu'on peut donc les assimiler à des « gated communities». Il va sans dire que leur accès se fait uniquement par automobile. L'aspect sécuritaire fait intégralement partie du standing du quartier, mais reste primordial puisque ces étrangers sont considérés comme « riches » et constituent des cibles potentielles pour les kidnappeurs. Ainsi sur la commune de Quezon city, ils ont intégré massivement les quartiers résidentiels de BF Homes (où résideraient 300 familles); c'est également le cas du village de Vista Real localisé à un kilomètre de là. 
Figure 4. Vista Real, une « gated community » prisée des Coréens sur la commune de Quezon City (août 2010).

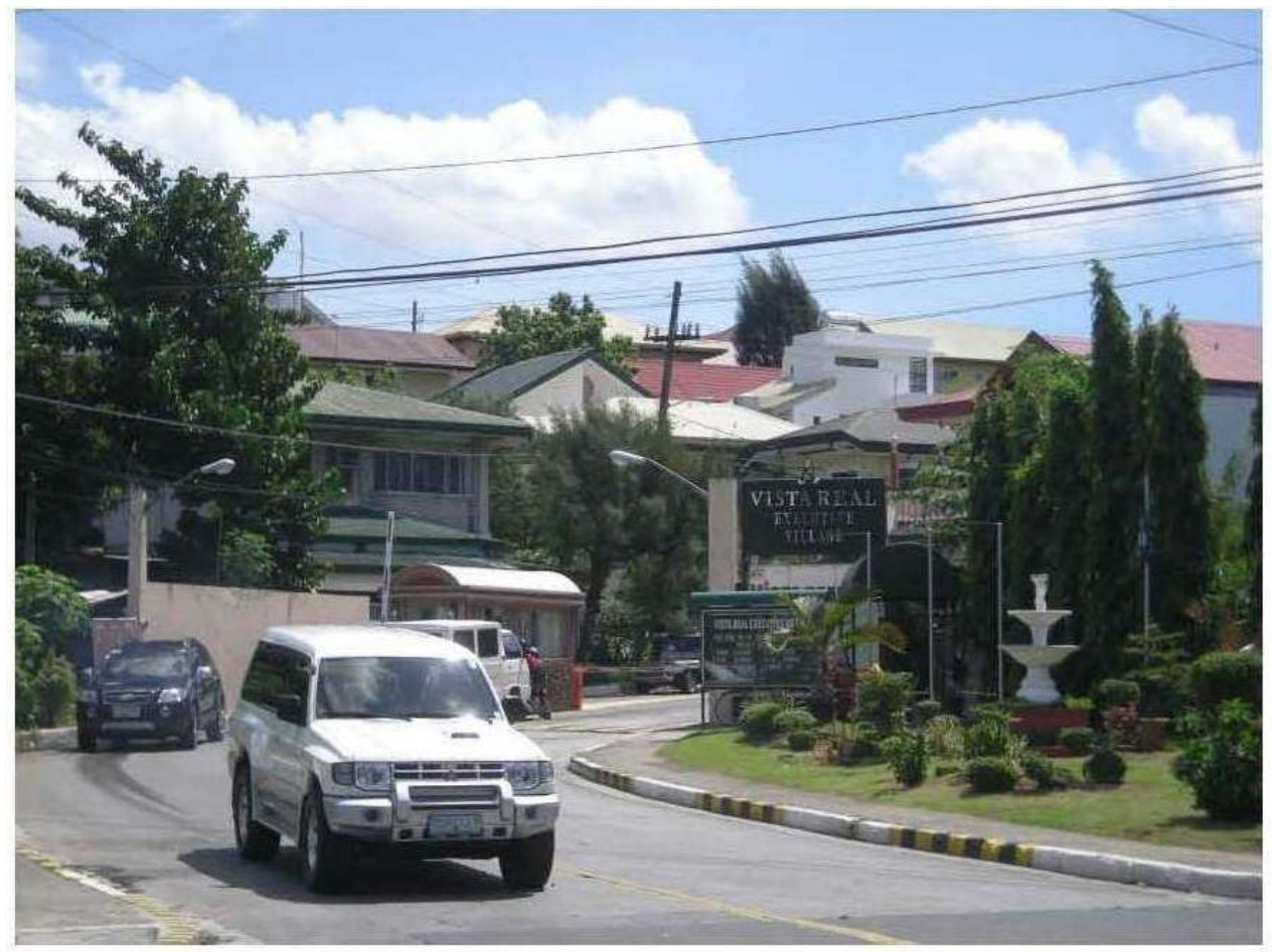

Figure 5. Les quartiers résidentiels dont les maisons deviennent des lieux de culte à Baguio (août 2010).

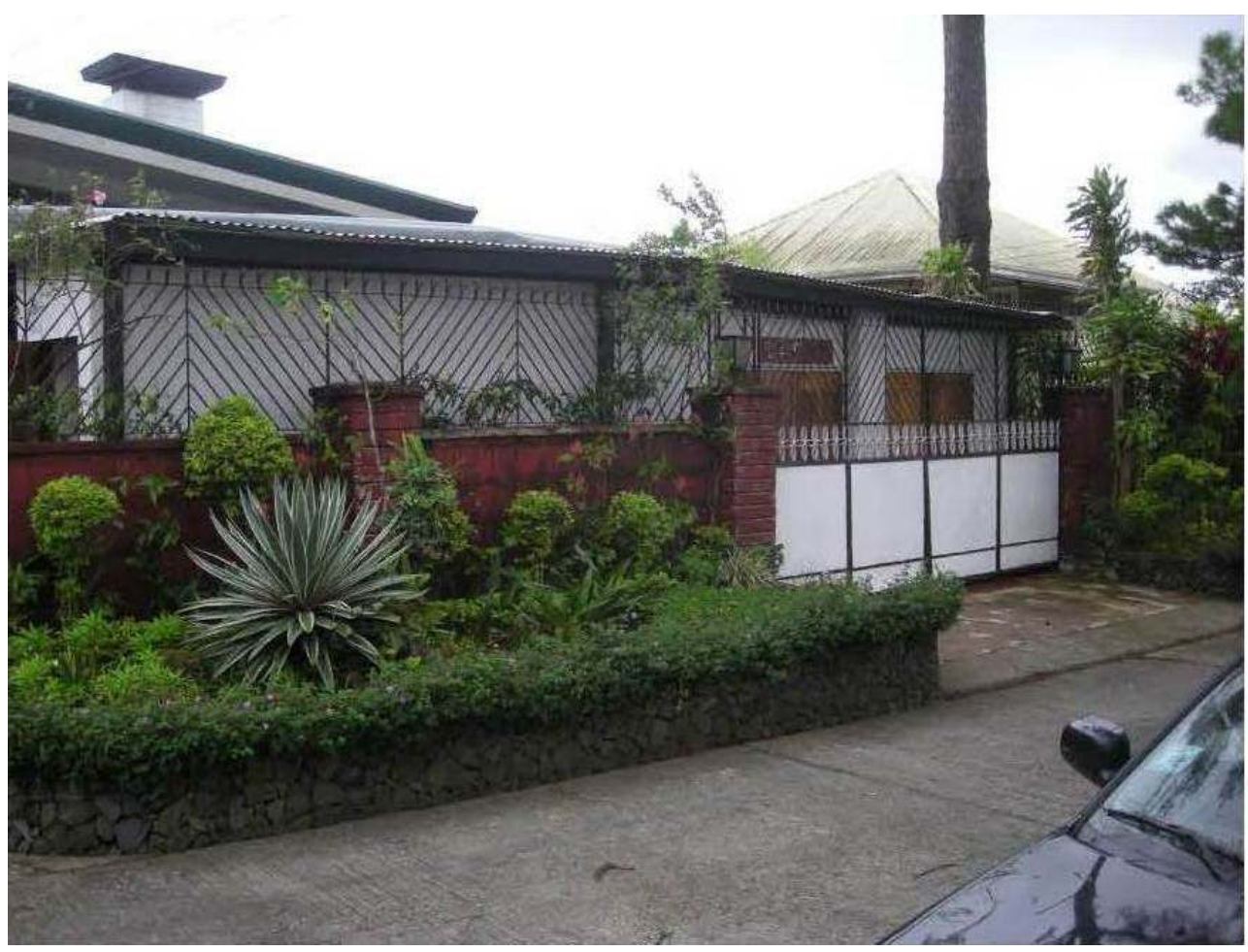

22 La ville reste le lieu privilégié d'implantation des Coréens mais ils investissent aussi des espaces situés « en marge » de la ville, où la nature est encore présente par des champs ou 
des espaces boisés. La présence de cours de golf à proximité des lieux de résidence complète cette envie de "grands» espaces. Ce descriptif de l'habitat résidentiel des Coréens nous confirme que ces migrants font partie des classes moyennes émergentes en quête d'un mode de vie différent de celui mené en Corée et que leur permet le coût de la vie dans leur nouveau pays d'accueil. On se doit de noter que certains employés ou cadres coréens des écoles de langue sur place habitent dans la structure de l'établissement.

La présence de ces résidants coréens transforme visuellement certaines rues. Ainsi, les axes routiers secondaires permettant d'accéder au quartier résidentiel de BF Homes (Quezon city) ou à des lieux incontournables de la communauté coréenne locale génèrant la création de commerces et de services de proximité. Ainsi les commerces les plus communément implantés sont des épiceries gorgées de produits importés, qu'il s'agisse de produits «secs » emballés ou de légumes. Dans des immeubles sans marquage visuel particulier s'installent des dentistes, coiffeurs au service de la communauté coréenne locale (le gérant est coréen et les employés sont philippins). Les établissements de restauration coréens sont également très présents dans un périmètre restreint. Ils sont dédiés à la population résidante mais aussi aux Coréens de passage. Les propriétaires de restaurants coréens en possèdent généralement plusieurs dans Metro-Manila ou ont ouvert un autre établissement dans un site touristique. Cette population de classe moyenne est aussi en quête d'un mode de vie à l'occidentale, ce qui explique la multiplication des enseignes de restaurant «au goût d'ailleurs» proposant une restauration italienne, ou de type brasserie à l'américaine.

La mobilité des Coréens est relativement réduite dans les endroits où ils séjournent: les étudiants évoluent dans la structure d'enseignement la journée et n'en sortent que le week-end pour aller à l'office dans la province de San Fernando/La Union pour ceux qui étudient à Baguio. Les Coréens sont encadrés et évoluent rarement seuls dans la ville : parlant difficilement anglais, ils sont continuellement encadrés et évoluent en groupe, d'autant plus si les séjours sont de deux ou trois semaines. On les retrouve alors dans le grand centre commercial «SM Mall» de Baguio où ils fréquentent assidument une boutique d'alimentation spécialisée (fig. 6).

Les Coréens construisent peu de bâtiments neufs pour leurs activités : ils occupent le tissu urbain existant et le rénovent. Certaines activités comme des écoles de langues ou des lieux de culte s'invitent dans des maisons individuelles; ces activités religieuses sont très discrètes dans le paysage urbain que ce soit à Baguio ou à Quezon city. Dans le cas de constructions neuves, le particularisme coréen n'apparaît pas : par exemple à Baguio une des écoles coréennes s'intitule « Union International School ». 
Figure 6. Les espaces de la présence coréenne dans Baguio.

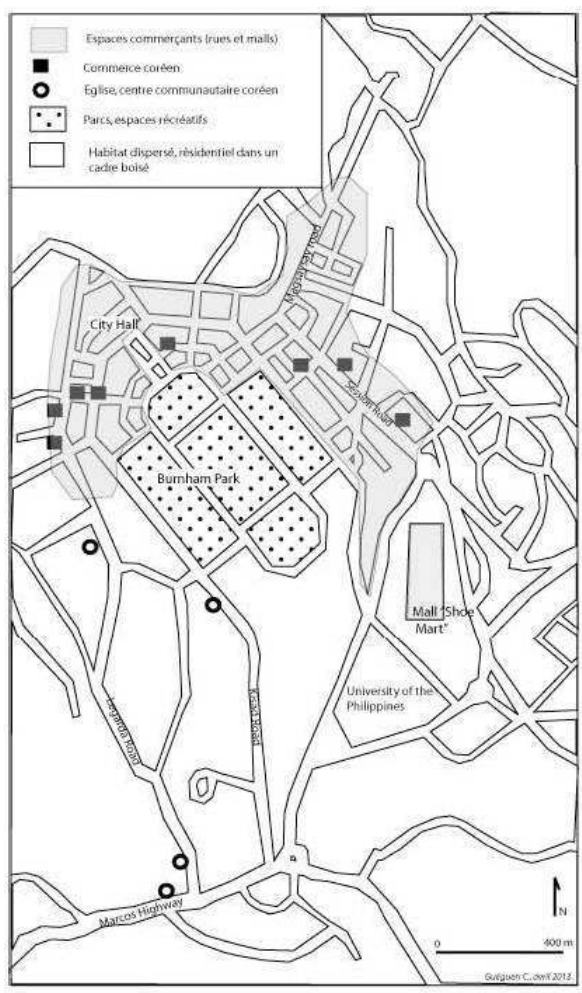

\section{Une présence coréenne au cœur de circulations spécifiques s'appuyant sur les « compétences » offertes par les Philippines}

\section{Des Coréens en quête de formations spécifiques promues par l'archipel}

L'offre de formation dans la capitale ou dans les grandes villes de province, qu'elle soit animée ou non par des entrepreneurs coréens, génère des mobilités touristiques spécifiques. En effet, les différents cursus universitaires proposant un enseignement totalement anglophone à des tarifs intéressants, les Coréens viennent donc aux Philippines pour une durée de quelques années le temps d'un parcours universitaire. D'autres y résident pour des séjours de courte durée afin de suivre des cours de langue anglaise uniquement et les écoles qui les accueillent sont des entreprises gérées par des résidents coréens. Les Coréens avaient déjà une expérience de l'apprentissage de l'anglais aux Philippines par l'intermédiaire de répétiteurs à distance employés par des services de cours de langue en ligne. Cet intérêt grandissant pour l'anglais est également dû au programme incitatif d'enseignement des langues voulu par le gouvernement du président Lee Myung-bak qui souhaitait faire de l'anglais non seulement la seconde langue du pays, mais aussi la langue de l'administration. De ce fait, les Coréens sont particulièrement intéressés par le B.A. mixte (Bachelor of Arts en Anthropologie, Anglais, Histoire, Sociologie et Conversation et Théâtre), le deuxième étant le B.S. en carrières sociales ( Bachelor in Science-Social Works). La population coréenne présente sur place est variée, 
composée d'étudiants, d'éducateurs, d'entrepreneurs et de missionnaires. Une faible proportion de ces Coréens constitue la population résidante permanente de la ville. Les villes de Cebu, Baguio et Dumaguete correspondent à ce profil. Quelle que soit la profession des adultes de la communauté, habitat et activité s'inscrivent dans un périmètre réduit.

La mise en avant des compétences linguistiques par les institutions philippines à l'étranger fait de l'archipel une destination de court et moyen séjour. Les Philippines adaptent leurs compétences en matière de formation aux Coréens qui souhaitent apprendre l'anglais ou réaliser un cursus en langue anglaise ${ }^{5}$. On se doit ici de rappeler le " passé commun » des Philippines et de la Corée du sud avec les Etats-Unis : quarante années de "protectorat américain» (1898-1940) ont fait des Philippines un Etat quasi anglophone et sensibilisé à la culture occidentale. Cette nouvelle offre est relayée abondamment par les services consulaires philippins de Corée. Les actions combinées des ministères du tourisme (Department of Tourism) et de l'emploi philippin (Department of Labour and Employment) se traduisent par des campagnes d'informations menées en Corée dans le cadre de salons spécialisés. L'apprentissage de l'anglais peut être proposé comme faisant partie d'un "package» comprenant des activités de découverte du pays (excursions, jeux....). Afin d'en faire la promotion, le Ministère du Tourisme Philippin participe à des conventions ou salons spécialisés. Le relais est assuré par les consulats philippins en Corée, lesquels renseignent et délivrent les visas.

De nombreux centres linguistiques ont vu le jour après autorisation des services concernés. Les Coréens sont à la base de la multiplication de ces centres d'apprentissage, ouverts en partenariat avec un associé philippin. Ainsi, à Baguio, 39 centres de langues ou de tutorat ont été répertoriés par la municipalité. Ils proposent trois types d'enseignement : des cours en ligne uniquement, des cours en ligne ou en direct et des centres d'hébergement et d'apprentissage (ce sont les plus grandes structures ${ }^{6}$ ).

Le centre de formation Monol implanté à l'extérieur de la ville est caractéristique de ce type de structure. Ainsi, depuis un an, l'école occupe un bâtiment de plusieurs étages qui héberge et instruit les jeunes Coréens en langue et civilisation anglo-saxonne. L'école occupait au préalable deux maisons qu'elle louait pour réaliser ses cours, mais avec l'augmentation du nombre d'étudiants, la structure a dû évoluer. Elle héberge désormais 280 étudiants qui séjournent entre 3 mois et 1 an, la moyenne du séjour étant de 6 mois. L'école fait de la publicité en Asie et attire des étudiants chinois, taiwanais, vietnamiens (5) et surtout des Coréens. 8 Coréens sont employés à temps plein à la direction de l'école ainsi que 10 Philippins. Certains des enseignants sont d'anciens « balikbayan » c'est-à-dire des Philippins ayant travaillé à l'étranger : ils mettent à profit leur expérience au compte des étudiants. Le profil des étudiants comprend de jeunes adultes et des professionnels. L'école recrute en plus des professeurs d'anglais, des instructeurs de fitness, de yoga, de pilates et d'aérobic pour assurer le bien-être des élèves le temps de cette formation intensive. L'ESL (English as a Second Language) est une véritable industrie, qui a débuté il y a une dizaine d'années. L'école emploie 250 personnes dont 150 enseignants. Certains centres n'ouvrent qu'une partie de l'année, lors des périodes de vacances coréennes, mais nombreux sont ceux qui sont peu sérieux et ne disposent pas des autorisations nécessaires. Tous les ans, les services de l'immigration démantèlent des écoles " sauvages » créées par des Coréens et dont le recrutement de stagiaires avait été réalisé à distance. En province, des extorsions de frais de scolarité prohibitifs ont été signalées. 
30 A Baguio, ville universitaire, $90 \%$ de la communauté coréenne sur place est composé d'étudiants, les personnes restantes sont des résidents adultes ou des missionnaires. La forte concentration d'étudiants coréens à Baguio s'explique aussi par le coût moindre de la vie en province ; ainsi pour 10000 pesos par mois (200 euros) l'étudiant parvient à se loger et se nourrir (pour Metro-Manila, le coût de la vie est supérieur d'environ 30 \%). Cet enseignement de l'anglais aux étrangers est devenu pour les établissements universitaires un vrai marché dont a pris conscience le gouvernement philippin: ce dernier l'a incorporé dans son programme touristique.

L'arrivée des étudiants est contrôlée par le Bureau de l'immigration ; ils arrivent avec un visa touristique, les démarches pour l'obtention d'un visa d'étude sont réalisées par le centre de formation. Le nombre d'étudiants coréens ayant obtenu ce visa a augmenté de 500 \% entre 2004 et 2008. En 2004, 5877 Sud-Coréens avaient obtenu le Special Study Permit , ils étaient 29155 en 2008. De plus, 13937 Sud-Coréens obtinrent un visa étudiant classique. Il faut cependant noter que pour les courts séjours beaucoup se contentent du visa touristique obtenu à leur arrivée. Depuis cinq ans, les Sud-Coréens constituent le groupe de nationalité étrangère le plus important aux Philippines. L'accréditation pour l'enseignement de l'ESL est délivrée par le Bureau de l'immigration et ces établissements deviennent uniquement des centres de langue pour étrangers. La durée des cours varie mais beaucoup optent pour un stage intensif de trois semaines; lequel permet d'asseoir des bases en grammaire et de conversation.

Ces centres de formation à Baguio, à Manille ou à Cebu permettent de valoriser d'une certaine manière l'expérience des Philippins ayant travaillé sous contrat dans les pays d'Asie anglophone (Singapour, Malaisie). On peut donc évoquer une forme de «Brain gain » minimal puisque seules les expériences linguistiques et civilisationnelles sont exploitées. Pour les enseignants philippins, le travail en ligne avec la Corée du sud est assez fastidieux bien qu'il n'y ait pas de décalage horaire car les enseignants travaillent de manière fragmentée (tôt le matin avant que les Coréens ne se rendent au travail ou le soir)

33 Ces centres linguistiques sont animés par des entrepreneurs coréens indépendants. Une des tendances globales pour les résidents permanents ou de longue durée, c'est-à-dire ceux qui ont transféré leur activité économique aux Philippines, est d'accéder à un autre mode de vie parfois plus aventureux en terme de sécurité de l'emploi mais surtout plus prestigieux grâce à un taux de change avantageux. Un taux de change défavorable peut aussi conditionner leur départ, comme ce fut le cas en 2008 (le won indexé au dollar américain avait perdu $30 \%$ de sa valeur). Il faut ajouter que le nombre de résidents coréens permanents est en baisse avec un peu plus de 88000 personnes par rapport à 2008 où ils étaient plus de 115 000. Pour quelques uns, il s'agit de quitter la modernité coréenne, une vie dédiée au «tout technologique» et s'engager dans une autre expérience.

\section{Des migrants coréens qui contribuent à une animation territoriale spécifique}

La création d'associations agit comme une force centripète, regroupe les Coréens qui maintiennent leur identité coréenne collectivement (Bergsten C.F. \& Inbom C. (eds), 2003). L'ancrage des populations coréennes aux Philippines s'appuie sur un ferment associatif entrepreneurial mais aussi religieux. On dénombre ainsi une cinquantaine 
d'associations protestantes implantées aux Philippines en plus de l'Eglise catholique coréenne. D'autres associations ont en charge la défense des intérêts professionnels de la communauté, une chambre de commerce coréenne a également vu le jour; les associations par secteur sont nombreuses (à titre d'exemple, la South Korean Import Association créée en 2002 a obtenu du gouvernement philippin la levée de l'interdiction des exportations de voitures d'occasion vers l'archipel) ou encore la United Korean Community Association créée en 1969, dont le but est de fédérer la communauté. Des médias communautaires ont vu le jour comme le Weekly Korea Post ou le Baguio Times qui est entièrement rédigé en coréen.

Pour les Sud-Coréens, les Eglises constituent la base de leurs réseaux sociaux, lesquels peuvent déboucher sur les structures d'enseignement comme c'est le cas à Dumaguete (Makil L., 2007). Le point de rencontre communautaire est l'église, qui est aussi le lieu des activités sociales de la communauté. Ces Eglises protestantes ou presbytériennes coréennes s'investissent auprès des populations pauvres des lieux et les centres de formation entretiennent quelques liens avec les pasteurs coréens de la ville. A Baguio, la Christian Methodist Church (CMC) occupe un bâtiment en rénovation effectuée par les volontaires de l'Eglise. Elle organise des séminaires et des rencontres entre les fidèles. Sept missionnaires coréens travaillent avec des Eglises philippines des alentours de Baguio. Un des projets repose sur la construction d'une église et la rémunération des pasteurs locaux. Le pasteur du lieu anime la messe quotidienne à 5 h30 du matin et compte 80 membres. Tous les membres des Eglises se rencontrent dans le cadre de la «Baguio Church Association" pour des rencontres inter-Eglises. Les structures religieuses sont particulièrement attentives aux étudiants présents dans la ville : la CMC organise un ramassage par mini-bus à leur attention.

Tableau 1. Eglises et personnels religieux coréens à Baguio en 2010 (Baguio Times, 2010).

\begin{tabular}{|l|l|}
\hline Eglises présentes à Baguio & Nombre de clerc(s) \\
\hline Korean Union Church/ Presbyterian Church & 4 \\
\hline Middle Nazareth Church/ Presbyterian Church & 1 \\
\hline Happy Church & 4 \\
\hline CMC Korean Church/ Méthodiste & 9 \\
\hline Vision Church/ Presbyterian & 2 \\
\hline Nazareth Church/ Prebyterian & 2 \\
\hline Beautiful, Peaceful Church/ Methodist & 2 \\
\hline Going all people/Baptiste & 1 \\
\hline Thanksful Church /Methodist & 3 \\
\hline
\end{tabular}

Ces Eglises ont une gestion distincte, enseignent la Bible à la seule communauté coréenne et organisent des activités de loisirs, sports et de retraite spirituelle. Certaines offrent un 
hébergement aux étudiants et des cours d'anglais. Les actions de mission sont réservées aux Philippins et certains programmes ciblent les jeunes (à titre de comparaison, en 2007, L. Makil dénombrait 5 Eglises à Dumaguete).

La présence des missionnaires et pasteurs coréens aux Philippines répond à deux attentes. Il s'agit d'abord d'établir une terre de mission en accord avec les filiales philippines des mouvements méthodistes ou presbytériens internationaux en s'appuyant sur des pasteurs locaux établis en zone rurale. Le second objectif s'inscrit dans la structuration de la communauté coréenne outre-mer : la présence d'un pasteur permet la recomposition de la structure sociale coréenne, dans laquelle les pasteurs ont une charge honorifique très importante. Bien que les Eglises soient regroupées autour de la « Korean Union Church ", une certaine concurrence existe entre ces dernières. Tous les Coréens ne se rassemblent pas autour d'une Eglise, et les activités de certains missionnaires sont décriées car ces derniers profiteraient de leur mission pour éduquer à moindre coût leurs enfants dans les meilleures écoles internationales de la ville.

Ces éléments importés aux Philippines génèrent une sociabilité coréenne à Baguio (restaurant, commerces, coiffeur, dentistes...) mais l'accès à des espaces réservés aux élites du lieu leur semble relativement fermé. En effet, il a été refusé à certains Coréens la carte de membre du club de golf de Baguio.

\section{Conclusions}

La présence des Coréens aux Philippines s'inscrit dans un processus mobilitaire multiscalaire. Ces populations coréennes pérennes ou intermittentes sur le sol philippin participent tout d'abord à la dispersion des communautés coréennes traditionnellement implantées dans des foyers où ces derniers sont déjà très nombreux, à l'image du Japon ou de la Chine. Ces déplacements géographiques ne concernent que les Coréens du sud dont le pays a renforcé ces partenariats économiques régionaux notamment dans le cadre de l'ASEAN. Les migrations et les déplacements de populations tout comme l'obtention des visas en sont facilités. Les résidents coréens sur place s'investissent largement dans ce parcours mobilitaire de formation encouragé par les autorités philippines en quête de diversification de leurs activités ; la manne touristique éducative doit aussi permettre de changer l'image du pays.

Cette mobilité est aussi permise par des transports bon marché et des structures d'enseignement régulées et encadrées (bien que des écoles illégales soient montées par des Coréens le temps des vacances scolaires). De manière plus générale, les Philippines jouent la carte d'un tourisme spécialisé et répondent à une demande "de proximité ", misant sur une nouvelle fonctionnalité des espaces en Asie orientale. On peut, dès lors, s'interroger sur la place des Philippines en tant que nouvel espace relais dans les migrations internationales. En effet, les étudiants coréens séjournent aux Philippines dans l'optique de partir vers un Etat anglophone. Se perfectionner en anglais peut leur permettre d'être plus intégré dans les administrations de Corée du sud. Les familles coréennes résidantes recherchent également un enseignement anglophone pour leurs enfants que l'on retrouve dans les écoles internationales du pays, offrant de ce fait un accès à des études universitaires anglophones avec l'obtention du baccalauréat international (IB). Les compétences de formation des Philippines sont désormais mises à disposition d'autres populations désireuses de participer à des flux migratoires 
internationaux. Résidents coréens et jeunes en formation se côtoient dans des territoires organisés et restreints, d'où la très forte impression de regroupements de ces populations quel que soit l'endroit, ce qui confirme le prolongement identitaire diasporique dans le secteur touristique. s'interroger sur leur pérennité résidentielle. La présence coréenne aux Philippines fluctue avec ampleur au gré de la valeur du won. Leur arrivée avait souvent été motivée par un différentiel avantageux. Ainsi la baisse de la monnaie coréenne et la crise économique mondiale ont généré de nombreuses pertes d'activités et notamment non seulement celles induites dans les services dédiés aux Coréens mais aussi dans la vente de produits auprès de la population philippine. De plus, la législation philippine concernant l'acquisition des biens fonciers et la création d'entreprise n'entrave pas les départs. En effet, il est impossible pour un étranger d'investir dans le foncier sauf dans de petits appartements, ce qui explique que les Coréens soient généralement locataires. La création d'une entreprise par un étranger ne se réalise qu'avec un partenaire local, si bien que les Coréens ne sont "propriétaires" de leur entreprise qu'à hauteur de $40 \%$. Quant aux professionnels étrangers, ils ne peuvent bien souvent pas travailler avec leurs diplômes sur place: c'est le cas des dentistes qui peuvent ouvrir un cabinet médical, mais qui doivent employer des dentistes philippins et gérer leur affaire avec un partenaire local. Les conditions foncières et professionnelles ne sont donc pas forcément favorables à l'emploi des Coréens et des étrangers en général sur place.

L'Etat philippin a su dès les années 1970 organiser des flux de main-d'œuvre vers l'extérieur. Aujourd'hui, il capte des populations désireuses d'apprentissages et les canalisent par une maîtrise des dispositifs mobilitaires multiformes impliquant largement les secteurs des transports et de l'immobilier, tout en permettant à la fois le réinvestissement des compétences acquises à l'étranger par les Philippins et la poursuite du lien diasporique indispensable à l'établissement de formes de tourismes coréennes sur place.

\section{BIBLIOGRAPHIE}

ABINALES AMOROSO D. (2005), State and Society in the Philippines, Rowman \& Littlefield, Langham, $351 \mathrm{p}$.

BERGSTEN C.F. \& INBOM C. (eds.) (2003), Korean in the world economy, Institute for international economics, special report 15, Washington, $180 \mathrm{p}$.

ADEY P. (2010), Mobility, Routledge, 267 p.

CORTES G., FARET L. (coord.) (2009), Les Circulations Internationales, Armand Colin, coll. U, Sc. humaines et sociales, $244 \mathrm{p}$.

CRESSWELL T., MERRIMAN P. (eds.) (2011), Geographies of mobilities : practices, spaces, subjects, Ashgate, 276 p. 
DEHOORNE L. (2002), « Tourisme, travail, migration : interrelations et logiques mobilitaires », Revue européenne des Migrations internationales, 18, 1, pp. 7-36.

FARET L. (2007), « Migrations internationales et développement à l'heure de la mondialisation », in CADÈNE P., La Mondialisation, L'intégration des pays en développement, SEDES, pp. 29-42.

MAKIL L.P. (2007), « South Koreans in Dumaguete : a preliminary study », in MIRALAO V.A., MAKIL L.P., Exploring Transnational communities in the Philippines, Philippine Social Science Council, pp. 49-57.

PARREÑAS R.S. (2003), Servants of globalization (Women, migration and domestic work), Ateneo de Manila University Press, Quezon city, 309 p.

PARREÑAS R.S. (2005), Children of global migration (transnational families and gendered woes), Ateneo de Manila University Press, Quezon city, 212 p.

PELLETIER P. (coord.) (2012), Géopolitique de l'Asie, Nouveaux continents, Nathan, 352 p.

RYANG S. \& LIE J. (eds.) (2009), Diaspora without homeland. Being Korean in Japan (Global, Area and international archive), University of California Press, 236 p.

TUGAUlT Y. (1984), « Les Coréens au Japon, Notes et documents », Population, 39, 6, pp. 1073-1075.

TYNER J.A. (2009), The Philippines, Mobilities, Identities, Globalization, Global realities series, Routledge, New-York \&London, 229 p.

Sites de quotidiens consultés :

www.inquirer.net, www.koreatimes.com

Site de l'ASEAN : www.asean.org

www.mofat.go.kr : données statistiques sur les communautés coréennes installées à l'étranger

\section{NOTES}

1. Statistiques du DOT (Department of Tourism), 2013.

2. Depuis août 2013, les touristes d'un grand nombre de pays peuvent séjourner aux Philippines 30 jours à compter de leur arrivée dans le pays (contre 21 jours auparavant).

3. On se doit d'ajouter que ces statistiques sont réalisées par les autorités locales à Boracay selon les enquêtes menées par les autorités administratives locales dans les aéroports et le port menant à Boracay, sachant que ce recensement n'est pas obligatoire, un nombre important de touristes n'y répond pas.

4. Cf établissement Alta Vista du groupe immobilier philippin DMCI.

5. Le processus de formation des Balikbayan, candidats philippins à l'émigration, a été bien décrit par Tyner J.A. (2009).

6. Enquête de terrain, août 2010. 


\section{RÉSUMÉS}

La population coréenne installée à l'étranger compte près de 7 millions de personnes ; $50 \%$ de cet effectif est implanté en Asie (surtout en Chine et au Japon). Les flux de populations coréennes en Asie se recomposent et se diversifient comme le montre leur implantation tardive aux Philippines. L'intérêt de cette étude est de comprendre les liens établis à plusieurs échelles pour permettre l'implantation de 88000 Coréens (2013) de manière pérenne et le million de déplacements touristiques opérés vers l'archipel philippin en 2012. L'inscription de ce phénomène est d'abord régionale avec l'intégration de la Corée du sud dans l'aire de mobilité de l'ASEAN mais il intègre aussi la spécificité philippine qui met à disposition ses compétences en matière de formation linguistique tout en permettant le prolongement culturel coréen par des réseaux entrepreneuriaux et sociaux indispensables à l'établissement des mobilités entre les deux Etats.

The Korean population settled overseas is composed of almost 7 millions; $50 \%$ of this group is based in Asia (mostly in China and Japan). The Korean migration waves in Asia evolve and become more diverse as shown by their late settlement in the Philippines. The main objective of this study is to understand the connections established in a multiscalar perspective to allow the establishment of 88000 Koreans permanently (2013) and the million of Korean tourists that reached the Philippines in 2012. The background of this phenomenon is, on the first hand regional with the integration of South-Korea in the ASEAN mobility perimeter, and on the other hand national as it takes into account the specificity of the Philippines that provide training in language education while allowing the establishment of a Korean cultural network through enterprises and social associations which are necessary to the mobility between the two countries.

\section{INDEX}

Keywords : The Philippines, South-Korean, ASEAN, mobilities, migrations, tourism, Air transportation

Mots-clés : Philippines, Sud-Coréens, ASEAN, mobilités, migrations, tourisme, transport aérien

\section{AUTEUR}

\section{CATHERINE GUÉGUEN}

Géographe, Espace, Nature et Culture (UMR 8185), catherinegueguen@hotmail.com 